Supporting Information

\title{
Ultralong Perovskite Microrods: One- versus Two-Step Synthesis and Enhancement of Hole-Transfer During Light Soaking
}

\author{
Xiangyang $\mathrm{Wu}$, Jin Wang and Edwin K. L. Yeow* \\ Division of Chemistry and Biological Chemistry, School of Physical and Mathematical \\ Sciences, Nanyang Technological University, 21 Nanyang Link, Singapore 637371 \\ Email: edwinyeow@ntu.edu.sg; Fax: +65 6791 1961; Tel: +65 63168759
}



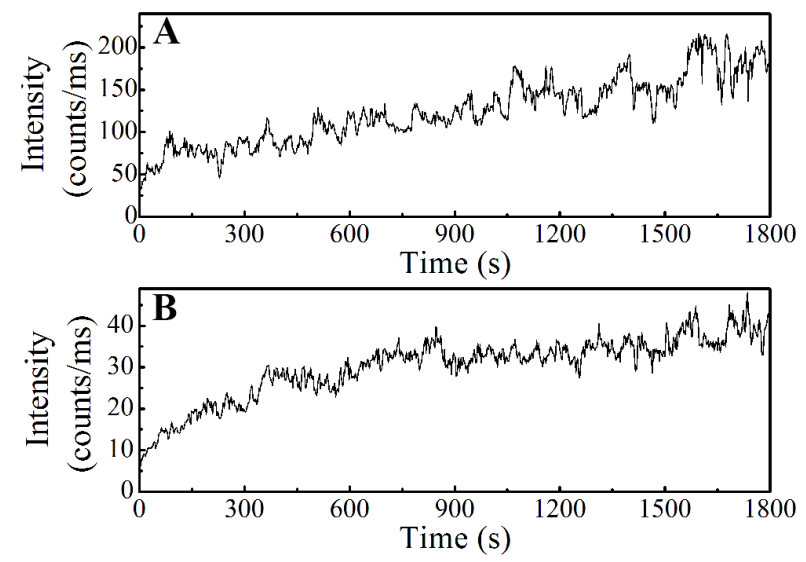

Figure S1. Typical PL intensity trajectories of two randomly selected points on $\mathrm{MAPbI}_{3}$ pervoskite microrods covered by a layer of PMMA. The $E F$ for (A) and (B) are 6.6 and 10.3, respectively. Bin-time is 2 s. 

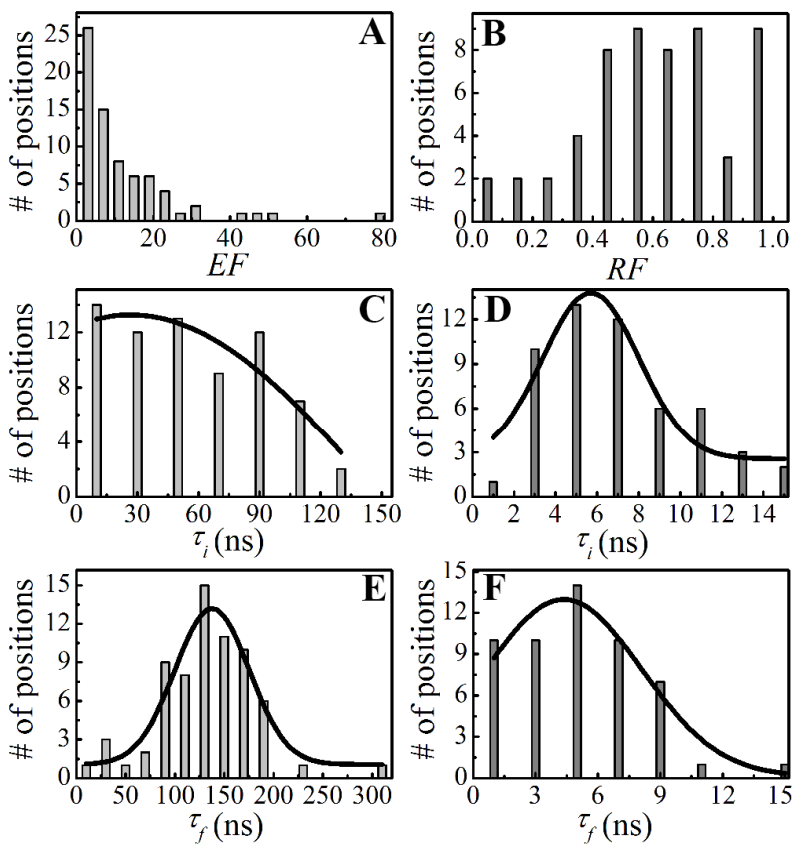

Figure S2. Histograms of the enhancement factor $(E F)$, reduction factor $(R F)$, initial lifetime $\tau_{i}$ (obtained from the monoexponential PL decays constructed from photons between 0 and 2 s) and final lifetime $\tau_{f}$ (obtained from the monoexponential PL decays constructed from photons between 1798 and $1800 \mathrm{~s}$ ) of several randomly chosen points on single pervoskite microrods prepared via the one-step synthesis method in the absence ((A) for $E F,(\mathrm{C})$ for $\tau_{i,}(\mathrm{E})$ for $\left.\tau_{f}\right)$ and presence ((B) for $R F$, (D) for $\tau_{i},(\mathrm{~F})$ for $\left.\tau_{f}\right)$ of spiro-OMeTAD. At the start of measurement, the mean values of $\tau_{i}$ in the absence and presence of spiroOMeTAD are ca. 26.6 (C) and $5.7 \mathrm{~ns}$ (D), respectively, which gives rise to $k_{h t} \sim 1.38 \times 10^{8} \mathrm{~s}^{-1}$. On the other hand, the hole-transfer rate calculated at the end of the light soaking measurement is $k_{h t} \sim 2.22 \times 10^{8} \mathrm{~s}^{-1}$, where the mean values of $\tau_{f}$ in the absence and presence of spiro-OMeTAD are $c a .137 .7$ (E) and $4.4 \mathrm{~ns}$ (F), respectively. 


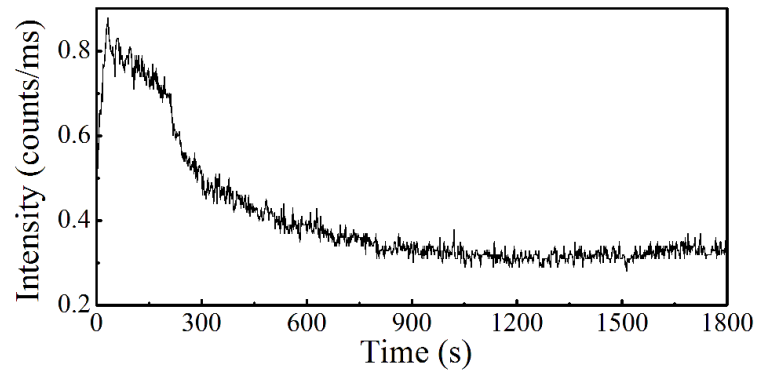

Figure S3. Typical PL intensity trajectory (bin-time $=2$ s) of a randomly selected point on an as-prepared $\mathrm{MAPbI}_{3}$ perovskite film in the presence of a layer of spiro-OMeTAD.

\section{Video captions:}

Video S1. Time-lapse microscopy images of the growth evolution of $\mathrm{PbI}_{2}$ microrods prepared by evaporating a droplet of $1 \mathrm{wt} \% \mathrm{PbI}_{2} / \mathrm{DMF}$ solution.

Video S2. Time-lapse microscopy images of the growth evolution of $\mathrm{PbI}_{2}$ microrods prepared by evaporating a droplet of $5 \mathrm{wt} \% \mathrm{PbI}_{2} / \mathrm{DMF}$ solution.

Video S3. Time-lapse microscopy images of the growth evolution of $\mathrm{MAPbI}_{3}$ perovskite microrods prepared via the one-step synthesis method. The growth of the perovskite is recorded by illuminating the sample with white light for the first $146 \mathrm{~s}$. The sample is then illuminated with laser light of excitation wavelength $=633 \mathrm{~nm}$, and at $t=9 \min 28 \mathrm{~s}$, PL from the perovskite is observed. The recordings from $t=2 \min 40 \mathrm{~s}$ to $9 \mathrm{~min}$ are not shown. 